\title{
0 uso do pool de plasma caseiro interfere no valor da RNI?
}

Primeira submissão em 05/12/11 Última submissão em 23/02/12 Aceito para publicação em 19/03/12 Publicado em 20/08/12

\section{Does the use of in-house pooled plasma interfere in the INR value?}

Paulo Henrique da Silva'; Janaína Risczik Arruda Correa²; Railson Henneberg ${ }^{3}$; Aguinaldo José do Nascimento ${ }^{4}$

unitermos

Tempo de protrombina

Relação normatizada internacional

Anticoagulante oral

\section{resumo}

Introdução: O tempo de protrombina (TP) é o teste de escolha para monitorar o nível de anticoagulação em pacientes que fazem uso de anticoagulantes orais. Nestes, atualmente, a anticoagulação é monitorada pelo valor da relação normatizada internacional (RNI). Objetivo: A finalidade deste trabalho foi determinar se um pool de plasma caseiro, com cinco (P5) e 20 (P20) amostras, pode ser usado como controle normal do TP e se o uso deste pool interfere no resultado da RNI. Material e métodos: Foram 32 dias de experimentos. Os dois pools de plasma caseiro foram analisados em relação a um controle normal de plasma comercial (PC). Para cada dia de experimento, fez-se o TP de P5, P20 e PC e também se determinou o valor de RNI para P5, P20 e PC em pacientes que fazem uso de anticoagulante oral. As ferramentas estatísticas utilizadas foram média $(X)$, análise de variância e teste de Tukey. Resultados: A análise estatística demonstrou que os valores do TP e da RNI não são significativamente diferentes para PC, P5 e P20. Conclusão: O pool de plasma caseiro pode ser utilizado como controle normal do TP e o seu uso não interfere no valor da RNI. abstract

Introduction: The prothrombin time (PT) test is commonly used to monitor anticoagulant levels in patients undergoing oral anticoagulant therapy. Currently, anticoagulation levels have been assessed through the international normalized ratio (INR) value. Objective: The objective of this study was to determine if inhouse preparations of polled plasma, containing five (P5) and 20 (P20) samples, respectively, may be used as normal control of PT and to assess its interference in INR values. Material and methods: The experiment was performed in 32 days. Both polled plasma preparations were analyzed in comparison with a commercial control (PC). PT was performed for PC, P5 and P20 daily and the INR value for PC, P5 and P20 was assessed in patients undergoing oral anticoagulant therapy. The applied statistical tools were mean value $(X)$, analysis of variance and Tukey test. Results: There were no statiscally significant differences in PT and INR for PC, P5 and P20. Conclusion: In-house polled plasma (P5 and P20) may be applied as normal control of PT and it does not interfere in the INR value.

\section{key words}

Prothrombin time

International normalized ratio

Oral anticoagulants 


\section{Introdução}

O tempo de protrombina (TP) foi desenvolvido por Quick, em 1935(12). É utilizado na clínica médica com três finalidades: (a) investigação de coagulopatias; (b) exame pré-operatório; (c) controle dos anticoagulantes orais. Tornou-se o teste de escolha para monitorar os anticoagulantes orais porque o TP inicia com ativação do fator VII6. O fator VII, para se tornar ativado, depende do ciclo da vitamina $\mathrm{K}$, o qual é inibido pelos anticoagulantes orais ${ }^{(10)}$. Após a anticoagulação, com anticoagulantes orais, o aumento do TP se dá, principalmente, pela redução nos níveis do fator VII. A atividade desse fator fica reduzida após 6 horas do início da anticoagulação; a do fator $\mathrm{X}$, após 40 horas; e a do fator II, após 60 horas ${ }^{(10)}$.

Um dos trabalhos mais relevantes para a anticoagulação oral foi o artigo publicado por Hull et al., em 1982, que demonstra que a tromboplastina obtida de cérebro de coelho, muito utilizada na América do Norte, é menos sensível à redução de atividade dos fatores dependentes de vitamina $\mathrm{K}$ que a tromboplastina utilizada em alguns países europeus ${ }^{(6)}$. Esse problema foi resolvido com a adoção de um sistema em que tromboplastinas comerciais são comparadas com tromboplastina de padrão internacional; para a qual se convencionou um índice de sensibilidade igual a 1,07. Essa comparação permite o cálculo de um índice de sensibilidade para tromboplastina comercial, o qual foi chamado de índice de sensibilidade internacional (ISI). O sistema de calibração das tromboplastinas comerciais (obtenção do ISI) é realizado pela World Health Organization $(\mathrm{WHO})^{(14)}$.

O ISI tem como finalidade padronizar a sensibilidade das tromboplastinas comerciais. A partir dele, é possível calcular a relação normatizada internacional (RNI), relação de tempos entre o TP do paciente e o TP do controle normal elevado ao valor do ISI. O monitoramento de indivíduos que utilizam anticoagulantes orais deve ser feito pela RNI ${ }^{(9,11,13)}$.

Henneberg et al., publicaram um trabalho, em 2011, acerca da avaliação do pool de plasma caseiro (composto por cinco e 20 amostras), como controle normal para o tempo de tromboplastina parcial ativada (TTP), e validou o uso desse pool como controle normal(3).

O objetivo deste trabalho foi utilizar o pool de plasma caseiro como controle normal do TP e avaliar se ele interfere no valor da RNI.

\section{Material e métodos}

Foram coletadas amostras de 337 pacientes em uso de anticoagulante oral, com idade variando entre 30 e 60 anos. As amostras foram coletadas pelo sistema a vácuo, usando como anticoagulante o citrato de sódio a 0,106 M. As amostras foram centrifugadas imediatamente após a coleta e o TP processado no prazo máximo de 4 horas.

O pool de plasma caseiro foi realizado com cinco (P5) e 20 (P20) amostras, segundo a técnica descrita por Henneberg et al.(3), e o pool de plasma comercial (PC) utilizado foi o da marca Siemens ${ }^{\circledR}$.

O TP foi realizado por meio do sistema de coagulação da Dade-Behring $\mathrm{BCS}^{\circledR}$ (Behring Coagulation System), que utiliza sistema ótico para processamento coagulométrico das amostras. O fotômetro faz leituras das amostras nos comprimentos de onda de 340,405 ou $570 \mathrm{~nm}$. O reagente utilizado (tromboplastina cálcica) foi o Tromborel $S$ da Siemens ${ }^{\circledast}$. O ISI da tromboplastina cálcica variou de 0,99 a 1,02.

Para a comparação dos resultados obtidos, foram realizadas duas análises estatísticas: a primeira comparou os valores do TP para PC, P5 e P20; a segunda comparou o valor da RNI utilizando PC, P5 e P20. Os dados foram avaliados utilizando-se as ferramentas estatísticas, média (X), análise de variância e teste de Tukey.

\section{Resultados}

A Tabela apresenta os valores do ISI e do TP com controle de PC, P5 e P20 para cada dia do experimento.

A Figura 1 mostra a análise estatística dos valores do TP para o PC, o P20 e o P5. A análise da variância e o teste de Tukey indicaram que não existe diferença estatisticamente significativa entre PC, P20 e P5.

A Figura 2 apresenta a análise estatística dos valores de RNI para PC, P20 e P5. A análise da variância e o teste de Tukey indicaram que não existe diferença estatisticamente significativa entre PC, P20 e P5.

\section{Discussão}

O efeito anticoagulante dos antagonistas da vitamina K é monitorado pelo TP, o qual é sensível à diminuição da concentração dos fatores dependentes de vitamina $\mathrm{K}^{(8)}$. Um 
Valores do ISI e do TP com o controle co-

Tabela 1 mercial, com pool de plasma P5 e P20.

\begin{tabular}{l|l|l|l|l|}
\hline Dia & ISI & TP (PC) & TP (P5) & TP (P20) \\
\hline
\end{tabular}$$
1
$$$$
2
$$

1,02

$$
12,7
$$

12,1

12

2

0,99

11,8

12

11,7

3

4

$$
0,99
$$

11,8

11,4

11,6

5

0,99

11,8

$11,5 \quad 11,4$

\begin{tabular}{l|l}
5 & 0,99
\end{tabular}

$$
11,8
$$

11,1

11

6

0,99

11,8

11,3

11,4

7

0,99

11,8

11,2

11,4

$\begin{array}{ll}7 & 0,99\end{array}$

11,8

11

11

9

0,99

11,8

11,3

11,3

10

0,99

11,8

11,4

11,4

11

0,99

11,8

11,7

11,9

12

0,99

11,8

11,6

11,6

13

1,02

12,7

12,1

12

14

0,99

11,8

\begin{tabular}{l|l}
12 & 11,8
\end{tabular}

15

0,99

11,8

11,6

11,8

16

0,99

11,8

12,2

12,2

17

0,99

11,8

12,1

12,4

18

0,99

11,8

11,5

11,5

19

0,99

11,8

11,8

12

20

0,99

11,8

11,6

11,6

21

0,99

11,8

12,2

12,2

22

0,99

11,8

12,1

12,4

23

0,99

11,8

11,3

11,4

24

0,99

11,8

11,4

10,8

25

0,99

11,8

11,8

12,1

26

0,99

11,8

11,9

11,8

27

0,99

11,8

11,7

11,7

28

0,99

11,8

11,5

11,6

29

0,99

11,8

11,8

11,4

30

0,99

11,8

11,4

11,3

31

0,99

11,8

12,1

11,8

32

0,99

11,8

11,4

11,5

ISI: índice de sensibilidade internacional; TP: tempo de protrombina; PC: plasma comercial.

componente crítico do TP é a tromboplastina utilizada, pois variações na composição desta influenciam no resultado do teste. Quanto menor a atividade da tromboplastina, menor será a variação do TP, e doses maiores do anticoagulante oral serão usadas para se atingir a dose terapêutica, predispondo o paciente a um risco maior de sangramento ${ }^{(5)}$.

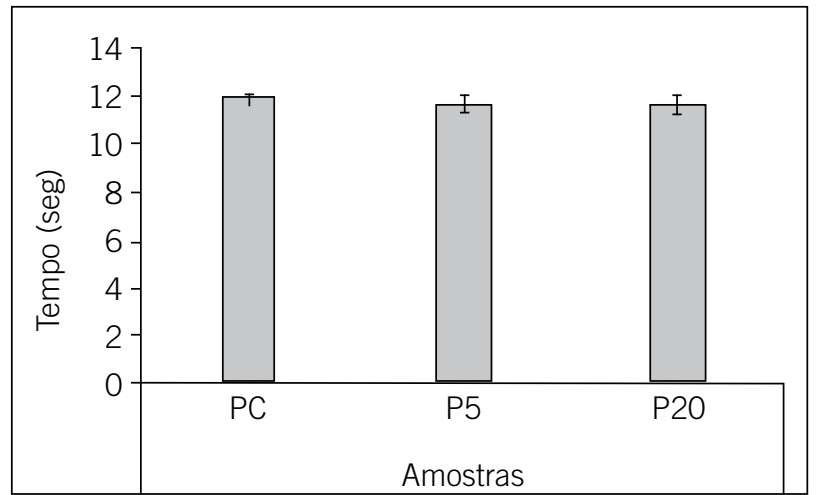

Figura 1 - PC: plasma comercial.

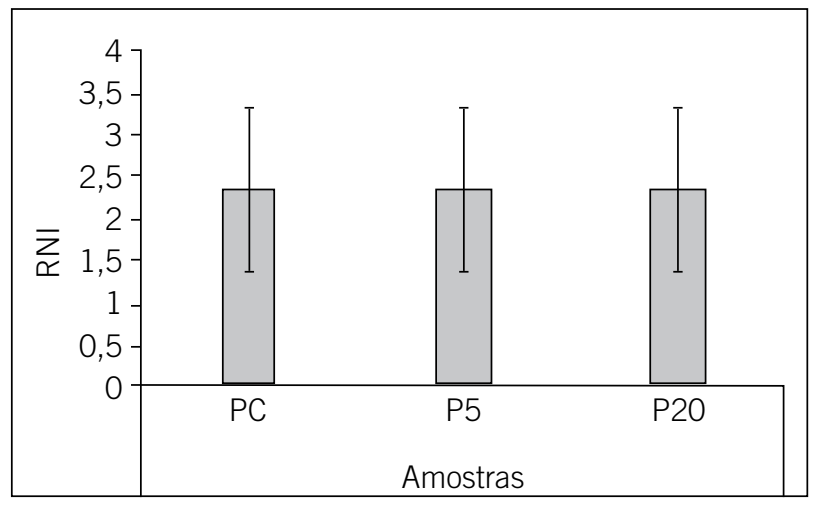

Figura 2 - RNI: relação normatizada internacional; PC: plasma comercial.

Os fabricantes de tromboplastina determinam a atividade de seu reativo pela obtenção do índice de ISI, que é usado como um fator de correção da atividade das tromboplastinas ${ }^{(8)}$. Estas, com alta atividade, tem ISI igual a 1. Na prática laboratorial, deve-se usá-las com ISI variando entre 1 a 1,4(5). A partir do ISI, é possível calcular a RNI, que melhorou bastante a padronização dos resultados do TP e, atualmente, é o parâmetro utilizado para monitorar a terapia com anticoagulantes orais ${ }^{(7)}$. Desse modo, os valores de RNI obtidos em diferentes laboratórios podem ser $\operatorname{comparados}^{(8)}$. No início da terapia, a RNI deve ser determinada a cada dois a três dias, por até duas semanas, até que a dose terapêutica seja atingida ${ }^{(8)}$. Um valor de RNI abaixo de 1,7 coloca o paciente em estado de hipercoagulabilidade; valores acima de 4,5 aumentam o risco de sangramento. Esse fato mostra a importância do rigor técnico na obtenção da RNI(1).

Mesmo com a adoção do RNI, ainda se tem dificuldades na padronização dos resultados. A precisão da RNI varia de acordo com a combinação reagente/instrumento e, além disso, cada laboratório deve estabelecer o valor do controle normal para o TP. O valor desse controle deve ser estabelecido utilizando as mesmas condições de coleta e técnica que foram usadas para a determinação do TP do paciente ${ }^{(12)}$. 
Quando o laboratório prepara o pool de plasma caseiro, este será representativo da população que o laboratório atende ${ }^{(14)}$ e é obtido a partir das mesmas condições de coleta e técnica do paciente que terá a RNI determinada. Nesse trabalho, fez-se a comparação estatística entre pool de PC e pool de plasma caseiro, com cinco e 20 amostras, e não houve diferença estatisticamente significativa. Isso demonstra que o pool de plasma caseiro, como descrito, pode ser utilizado na obtenção do controle normal para o TP e está de acordo com a literatura ${ }^{(2,15)}$.
Outra pergunta que este trabalhou procurou responder é se o uso do pool de plasma caseiro interfere no valor da RNI. Essa é uma questão crucial, visto que a janela terapêutica dos antagonistas da vitamina K é muito estreita. Ficou demonstrado que não há diferença estatística no valor da $\mathrm{RNI}$, quando se compara o pool de plasma caseiro (com cinco e 20 amostras) com o PC.

O trabalho conclui que o pool de plasma caseiro (com cinco e 20 amostras) pode ser utilizado como controle normal do TP e, quando utilizado para o cálculo da RNI, não interfere no resultado.

\section{Referências}

1. ALBERS, G. W.; DALEN, J. E.; LAUPACIS, A. Antithrombotic therapy in atrial fibrillation. Chest, v. 119, Suppl. 1, p. 194S-206S, 2001.

2. BARRANTES, A. B. El uso de reactivos estandarizados y el control de calidad en el método para el tiempo de protrombina. Ver Costar Cien Med, v. 3, p. 41-50, 1982.

3. HENNEBERG, R. et al. Avaliação do poolde plasma caseiro como controle normal para o tempo de tromboplastina parcial ativada (TTPa). J Bras Patol Med Lab, v. 47, v. 1, 2011.

4. HIRSH, J. Oral anticoagulant drugs. N Engl J Med, v. 324, n. 26, p. 1865-75, 1991.

5. HOFFMAN, R. et al. Hematology: basic principles and practice. 4. ed. Oxford: Elsevier, 2005.

6. HULL, R.; HIRSH, J.; JAY, R. Different intensities of anticoagulant therapy in the long term treatment of proximal-vein thrombosis. N Engl J Med, v. 307, p. 1676-81, 1982.

7. JOHNSTON, M.; HARRISON, L.; MOFFAT, K. Reliability of international normalized ratio for monitoring the induction phase of warfarin: comparison with the prothrombin time ratio. J Lab Clin Med, v. 128, p. 214, 1996.
8. KAUSHANSKY, K. et al. Williams hematology. 8. ed. Nova lorque: McGraw Hill, 2011.

9. KAZAMA, M.; SUZUKI, S.; ABE, T. Evaluation of international normalized ratios by a controlled Field survey with 4 different thromboplastin reagents. Thromb Haemost, v. 64, p. 535-41, 1990.

10. LOSCALZO, J.; SCHAFER, A. I. Thrombosis and hemorrhage. 2. ed. Baltimore: Williams \& Wilkins, 1994.

11. National Committee for Clinical Laboratory Standards NCCCLS H28-P. Proposed guidelines for the one-stage prothrombin time test (PT). Villanova: NCCLS, 1980.

12. QUICK, A. J.; STANLEY-BROWN, M.; BANCROFT, F. W. A study of the coagulation defect in hemophilia and in jaundice. Am J Med Sci, v. 190, p. 501-11, 1935.

13. RAY, M. J.; SMITH, I. R. The dependence of the International Sensitivity Index on the coagulometer used to perform the prothrombin time. Thromb Haemost, v. 63, p. 424-9, 1990.

14. SILVA, P. H.; HASHIMOTO, Y.; ALVES, H. B. Hematologia laboratorial. Rio de Janeiro: Revinter, 2009.

15. STIENE- MARTIN, E. A.; LOTSPEICH-STEININGER, C. A.; KOEPKE, J. A. Clinical hematology: principles, procedures, correlations. 2. ed. Philadelphia: Lippincott, 1998. 